\title{
The Impact of Discharged Loop Diuretic Dose to Home Dose on Hospital Readmissions in Patients with Acute Decompensated Heart Failure: A Retrospective Cohort Study
}

\author{
Mohannad Alshibani, Pharm.D, BCPS, ${ }^{1,2}$ Samah Alshehri, Pharm.D, MS, BCPS, ${ }^{1,2}$ \\ Wejdan Alyazidi, Pharm.D Candidate, ${ }^{3}$ Asmaa Alnomani, Pharm.D Candidate, ${ }^{3}$ Ziyad Almatruk, Pharm.D, ${ }^{1}$ \\ Tahani Almeleebia, Pharm.D, BCPS, BCACP ${ }^{4}$ \\ ${ }^{1}$ Faculty of Pharmacy, Department of Pharmacy Practice, King Abdulaziz University, Jeddah, Saudi Arabia; ${ }^{2}$ College of Pharmacy, \\ University of Arizona, Tucson, Arizona, USA; ${ }^{3}$ Faculty of Pharmacy, King Abdulaziz University, Jeddah, Saudi Arabia; ${ }^{4}$ College of \\ Pharmacy, Department of Clinical Pharmacy, King Khalid University, Abha, Saudi Arabia
}

\section{ABSTRACT}

Background: Acute decompensated heart failure (ADHF) is associated with a high rate of hospital readmission. The aim of this study is to examine the effect of the discharge diuretic dose compared with the home diuretic dose on hospital readmission in patients with ADHF.

Methods: A single center retrospective cohort study included patients with a confirmed diagnosis of ADHF with an ejection fraction of less than $40 \%$. The sample was divided in two groups. The first group received a total daily discharge diuretic dose that was greater than the home dose; the second group received a daily discharge diuretic that was equal to or less than the home dose. The primary outcome was all-cause 30 -day readmission rate. The secondary outcomes were all-cause 60-day and 90-day readmission rates.

Results: A total of 206 patients met inclusion criteria; 117 patients received a higher loop diuretic dose at discharge, while 89 were discharged with a loop diuretic that was equal to or less than the home dose. Patients in the increased-dose group had an allcause 30 -day readmission rate of $20.5 \%$ compared with $37.1 \%$ of patients with equal or reduced-dose group; $P=.007$. Additionally, there were lower readmission rates in 60 and 90 days between the increased and equal or reduced groups (33.3\% versus $52.8 \%$, $P<.017$, and $41.0 \%$ versus $62.9 \%, P<.003$, respectively.

Conclusions: Among patients admitted to hospital with ADHF and reduced ejection fraction, a discharge loop diuretic dose higher than the home dose was associated with decreased all-cause 30-day, 60-day, and 90-day readmission rates.

\section{INTRODUCTION}

Heart failure (HF) is a common clinical disorder resulting from any structural or functional impairment of ventricular

Received April 19, 2020; accepted fune 8, 2020.

Correspondence: Mohannad Alshibani, PharmD, BCPS, Assistant Professor, Department of Pharmacy Practice, King Abdulaziz University, Faculty of Pharmacy, Feddah, PO Box 22252; +966126400000/21146; fax +966126951696 (e-mail: malshibani@kau.edu.sa). filling or ejection of blood [Go 2013]. A 2013 update from the American Heart Association (AHA) showed that there were 5.1 million individuals with HF in the United States in 2006, and the prevalence continues to increase, especially for individuals greater than 65 years of age, who will make up one-fifth of the American population by 2050 [Yancy 2013]. The high rate of HF readmission occurs due to long-term medication regimen complexity and the difficulty of weight management [U.S. Department of Health and Human Services 2011; Jovicic 2006]. Patients hospitalized for HF are at a very high risk of readmission, with a 1 -month readmission rate of 25\% [Krumholz 2009]. In the Organized Program to Initiate Lifesaving Treatment in Hospitalized Patients with Heart Failure (OPTIMIZE-HF), the rate of hospital readmissions at 60-90 days was around 30\% [Fonarow 2007]. According to the Saudi Heart Association, the average age to develop heart disease in Saudi Arabia is 56 years, which is 10 years younger than the global average. Heart disease is associated with $42 \%$ of Saudi Arabia's non-communicable disease deaths in 2010 [Arab News 2018; MOH News 2013].

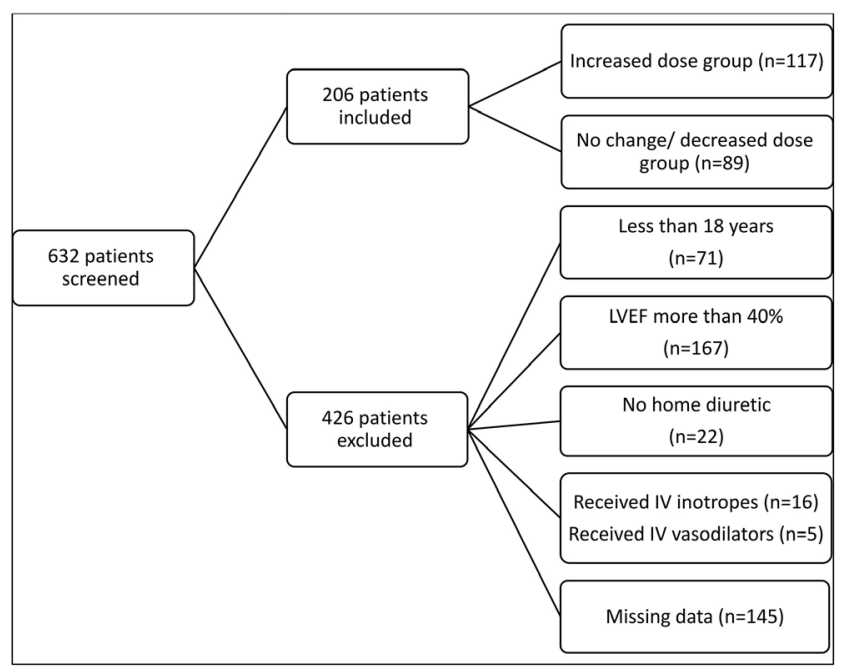

Flow of patients throughout the study 
Table 1. Baseline characteristics

\begin{tabular}{|c|c|c|c|}
\hline Age (year), mean $( \pm S D)$ & $63.1(12.7)$ & $61.9(14.4)$ & .55 \\
\hline Female, n (\%) & $33(37.1)$ & $44(37.6)$ & \\
\hline Height $(\mathrm{cm})$, mean $( \pm \mathrm{SD})$ & $161.2( \pm 15.8)$ & $160.4( \pm 20.2)$ & .77 \\
\hline Saudi patients, n (\%) & $29(32.6)$ & $49(41.9)$ & .173 \\
\hline Non-Saudi patients, n (\%) & $60(67.4)$ & $68(58.1)$ & \\
\hline Serum creatinine (umol/L), (median, IQR) & $120(80-150)$ & $130(90-174)$ & .954 \\
\hline Creatinine clearance (ml/min), (median, IQR) & $51.0(37.9-82.4)$ & $54.8(31.1-98.5)$ & .78 \\
\hline NTproBNP (pg/ml), (median, IQR) & $6182(1704-17010)$ & $3316(1578-11291.5)$ & .08 \\
\hline Diabetes mellitus, $\mathrm{n}(\%)$ & $62(69.7)$ & $71(59.7)$ & .18 \\
\hline Atrial fibrillation, $\mathrm{n}(\%)$ & $25(28.1)$ & $28(23.9)$ & .49 \\
\hline Stroke, TIA, n (\%) & $13(14.6)$ & $11(9.4)$ & .25 \\
\hline ACEls/ARBs, $\mathrm{n}(\%)$ & $86(96.6)$ & $110(94.1)$ & .39 \\
\hline BBs, n (\%) & $78(87.6)$ & $103(88.0)$ & .93 \\
\hline Hydralazine/ISDN, n (\%) & $51(57.3)$ & $66(56.4)$ & .89 \\
\hline Aldosterone antagonists, n (\%) & $67(75.3)$ & $83(70.9)$ & .49 \\
\hline Total home diuretic dose (mg), (median, IQR) & $80(40-120)$ & $40(40-80)$ & $<.001$ \\
\hline
\end{tabular}

$\mathrm{SD}=$ standard deviation; $\mathrm{n}=$ number; $\mathrm{cm}=$ centimeter; $\mathrm{Kg}=$ kilogram; NTproBNP = N-terminal pro b-type Natriuretic Peptide, TIA= transient ischemic attack; $\mathrm{ACE}=$ angiotensin converting enzyme inhibitor; $\mathrm{ARB}=$ angiotensin receptor blocker; $\mathrm{BB}=$ beta blocker; ISDN= isosorbide dinitrate

Acute decompensated heart failure (ADHF) is a new-onset or exacerbating HF, frequently leading to hospitalization or a visit to the emergency department (ED). ADHF is associated with a very high risk of hospital readmission, which can be as high as $50 \%$ at 6 months, and 1-year mortality is around 30\% [Kociol 2010]. The goals of treatment in ADHF patients are hemodynamic and oxygen stabilization and symptom relief. The best management to relieve fluid overload in patients with ADHF is the use of an intravenous (IV) loop diuretics without delay because early intervention has been associated with better outcomes [Kannel 2000]. Several IV diuretics, including furosemide, bumetanide, and torsemide, are recommended for ADHF. Vasodilators and inotropes may be needed as well in some cases to manage elevated filling pressures and/or left ventricular afterload [Publication Committee for the VMAC Investigators 2002; Felker 2011; Brater 1984; Brater 2011].

Although loop diuretics play a crucial role in patients with chronic and acute decompensated HF by producing rapid relief of symptoms and decreasing morbidity, they do not improve survival. The recent heart failure guidelines recommend an initial dose that is higher than or equal to the home dose of loop diuretics in patients with ADHF [Yancy 2013]. Accordingly, IV diuretics should be individualized and titrated depending on several factors that include the maintenance total daily oral dose, renal function, severity of fluid overload, and diuretic resistance. Patients treated with loop diuretics for a long time might need a higher dose of IV loop diuretics. Once the patient's symptoms are reduced, IV loop diuretics must be converted to an effective oral dose before discharge [Felker 2011; Brater 1984; Brater 2011]. However, optimal discharge oral dose is a source of debate since each patient may respond to IV diuretics, during admissions in different ways, which might affect the transition to a proper oral discharge dose.

Therefore, the aim of this study is to evaluate the effect of discharge diuretic dose compared with the home diuretic dose on hospital readmissions in patients admitted with ADHF. We hypothesize that a discharge loop diuretic dose that is higher than the home dose is associated with fewer readmissions. 
Table 2. Main outcomes

\begin{tabular}{lccc}
\hline Variable & Lower or equal dose $(\mathrm{N}=89)$ & Higher dose $(\mathrm{N}=117)$ & $P$ \\
\hline 30-day readmission, $\mathrm{n}(\%)$ & $33(37.1)$ & $24(20.5)$ & .008 \\
60-day readmission, $\mathrm{n}(\%)$ & $47(52.8)$ & $39(33.3)$ & .005 \\
90-day readmission, $\mathrm{n}(\%)$ & $56(62.9)$ & $48(41.0)$ & .002
\end{tabular}

\section{METHODS}

This is a single center retrospective cohort study conducted in a tertiary teaching hospital with a total capacity of 1,002 beds. Data were retrieved through medical records for all patients admitted to the ED with a primary diagnosis of ADHF from January 1, 2016 to December 31, 2018. This study was approved by the Institutional Review Board (IRB) of King Abdulaziz University Hospital (KAUH), Jeddah, Kingdom of Saudi Arabia (KSA).

Patients were included in the study if they: (1) were 18 years of age or older with a confirmed diagnosed of ADHF with an ejection fraction of less than $40 \%$; (2) were on loop diuretics preceding their admission; and (3) were discharged on loop diuretics. Patients were excluded if they: (1) received any IV inotropes during their hospital stay; (2) received any IV vasodilators during their hospital stay; (3) had ejection fraction of more than $40 \%$; (4) died during their hospital stay; or (5) had missing information.

Patients were classified into two groups, according to their total daily loop diuretic dose at discharge in relation to their home dose prior to admission. The first group received a total daily discharge diuretic dose that was higher than the home dose, while the second group received a total daily discharge diuretic dose that was equal to or less than the home dose. The primary outcome was all-cause readmission within 30 days of discharge, while secondary outcomes were all-cause readmission rates within 60 and 90 days.

Data collection: Patient demographics, including age, gender, nationality, height, and weight were collected. The presence of cardiovascular-related comorbidities, including atrial fibrillation, diabetes, history of transient ischemic attack/stroke, coronary artery disease (CAD), and acute kidney injury (AKI) also were collected. AKI was defined as a rise in serum creatinine $>0.5 \mathrm{mg} / \mathrm{dL}$ during hospitalization. Laboratory data, including serum creatinine and N-terminal pro b-type natriuretic peptide (NTproBNP), were obtained during admission. Additionally, information about other HF medications, including angiotensin-converting enzyme inhibitors (ACEIs), angiotensin receptor blockers (ARBs), beta-blockers, aldosterone antagonists, and isosorbide dinitrate (ISDN) plus nitrate, were collected at discharge as well.

Data analysis: Using the Statistical Package for the Social Sciences (SPSS) version 21, the data was entered and analyzed. Qualitative variables were described in frequencies and percentages, while quantitative data were expressed in means \pm standard deviations (SD) for the normally distributed variables and median (interquartile range IQR) for the non-normally distributed variables. For baseline characteristics, we used Chi-Square test, unpaired Student T test, and Mann-Whitney test to compare the two groups. A Chi-square test was used to evaluate the outcomes of the study. A twotailed $P$-value of less than 0.05 was considered significant. To detect a $20 \%$ difference in primary outcome between the two groups with $80 \%$ power and a significance level of $5 \%$ (twosided), a total sample size of 206 patients would be required in our study.

\section{RESULTS}

A total of 632 patients were screened for eligibility, and 206 patients met the inclusion criteria and were included in the study. Of the 632 screened patients, 426 were excluded from our study for the following reasons: 71 patients were less than 18 years old; 167 patients had ejection fraction of more than $40 \%$; 22 patients were not on diuretics at home; 16 patients received IV inotropes during hospital stay; five patients received IV vasodilators during hospital stay; and 145 patients had missing information (Figure). Of the 206 included patients, 117 patients were discharged with a loop diuretic dose that was higher than the home dose prior to admission (increased-dose group), and 89 patients were discharged with a loop diuretic does equal to or less than the home dose prior to admission (equal or reduced-dose group).

There was no significant difference between the two groups in terms of age, gender, height, weight, and nationality. The median total daily diuretic doses in the increaseddose group were $40 \mathrm{mg}(\mathrm{IQR}=40$ - 80) before admission, $240 \mathrm{mg}(\mathrm{IQR}=160$ - 320) during hospitalization, and $120 \mathrm{mg}$ $(\mathrm{IQR}=80-160)$ at discharge. On the other hand, the median total daily diuretic doses in the equal or reduced-dose group were $80 \mathrm{mg}$ daily $(\mathrm{IQR}=40$ - 120) before admission, $240 \mathrm{mg}$ (IQR=160 -340) during hospitalization, and $80 \mathrm{mg}$ (IQR = $40-80)$ at discharge. At the time of hospital discharge, there was no significant difference between the increased-dose and equal or reduced-dose groups in the rate of prescribing ACEIs/ARBs (94.1\% versus 96.6\%), beta blockers $(88.0 \%$ versus $87.6 \%$ ), ISDN /hydralazine (56.4\% versus $57.3 \%)$, and aldosterone antagonists (70.9\% versus $75.3 \%)$ (Table 1$)$.

Furthermore, there was no significant difference between the increased-dose and equal or reduced-dose groups in the presence of comorbidities, including coronary artery disease $(36.8 \%$ versus $30.3 \%)$, diabetes mellitus $(59.7 \%$ versus $69.7 \%)$, atrial fibrillation (23.9\% versus $28.1 \%$ ), and stroke/ TIA $(9.4 \%$ versus $14.6 \%)$. The median ejection fraction was 
$30 \%$ (IQR 21-35) in the increased-dose group and 30\% (IQR $20-35)$ in the equal or reduced-dose group $(P=.60)$. The increased-dose group had a median NTproBNP level of 3316 $\mathrm{pg} / \mathrm{ml}$ (IQR=1578-11291), and the equal or reduced-dose group had a median NTproBNP level of $6182 \mathrm{pg} / \mathrm{ml}$ (IQR $=1704-17010)(P=.08)$.

There was no significant difference between the increaseddose and equal or reduced-dose groups with regard to the incidence of AKI, during the hospital stay $(15.4 \%$ versus $11.2 \%)$. Additionally, median serum creatinine levels were $130 \mathrm{umol} / \mathrm{L}(\mathrm{IQR}=90-174)$ and $120 \mathrm{umol} / \mathrm{L}(\mathrm{IQR}=80-150)$, respectively, while the median creatinine clearance during hospital stay was $54.8 \mathrm{~mL} / \mathrm{min}(\mathrm{IQR}=31.1-98.5)$ and 51.0 $\mathrm{mL} / \mathrm{min}(\mathrm{IQR}=37.9-82.4)$, respectively.

Main outcomes: Regarding the primary outcome, the 30 -day readmission rate was $20.5 \%$ in the increased-dose group compared with $37.1 \%$ in the equal or reduced-dose group $(P=.008)$. Moreover, 60 -day and 90 -day readmission rates remained significantly lower in the increaseddose group compared with the equal or reduced-dose group (33.3\% versus $52.8 \% ; P=.005)$ and $(41.0 \%$ versus $62.9 \%$; $P=.002$ ), respectively (Table 2 ).

\section{DISCUSSION}

Diuretics are the cornerstone of treatment of volume overload in patients with HF. The use of IV loop diuretics in $\mathrm{ADHF}$ is commonly required for most patients to achieve rapid diuresis and improve symptoms. The proper dosing of diuretics in ADHF can be driven by several factors, which can include kidney function, severity of fluid overload, prior diuretic dose at home, and diuretic resistance [Hasselblad 2007]. The recent heart failure guidelines recommend initiating a loop diuretic dose at discharge that is higher or equal to the home dose in patients with acute decompensated heart failure [Yancy 2013]. This study highlighted the impact of the total discharged diuretic dose, when compared with the total home diuretic dose in patients with ADHF on hospital readmissions.

The key finding of our results shows that patients who received a discharge diuretic dose that was higher than the home dose had a 30-day readmission of $20.5 \%$, compared with $37.1 \%$ if they were discharged with a dose that was equal to or less than the home dose. This finding is consistent with what was reported by Woodruff et al [Woodruff 2016]. They stated that patients in the increased-dose group had an all-cause 30 -day readmission rate of $20 \%$ compared with $38 \%$ of patients in the equal or reduced-dose group $(P=.0009)$. This consistency in both studies emphasizes the benefit of increasing diuretic dose at discharge to decrease hospital readmissions.

The reduction in hospital readmissions remains significant at 60 and 90 days. To the best of our knowledge, this is the first study to examine the effect of increased loop diuretics in ADHF on 60-day and 90-day readmissions. This finding confirms that the benefit of increasing the outpatient loop diuretics dose will continue up to 90 days after discharge.
It should be noted that most of patients enrolled in the equal or reduced-dose group received a discharge diuretic dose that was equal to the home dose (77 patients, $86.5 \%$ ), which is consistent with guideline recommendations. However, the remaining patients (12 patients, $13.5 \%$ ) received a lower dose. One explanation might be the inconsistency of the medication reconciliation between what is seen in pharmacy records and physician records. Another explanation is that some patients might have been informed by their physicians that they received a lower dose at home than what was reported in the hospital system. This might be true in some patients, who had some instructions to reduce their diuretic dose if their weight (volume status) was optimal.

Another point to consider is that most of enrolled patients in our study were receiving the recommended maintenance therapy for $\mathrm{HF}$ with reduced ejection fraction (HFrEF). The recent guidelines recommend beta blockers, ACEIs or ARBS, angiotensin receptor-neprilysin inhibitors (ARNIs), and aldosterone antagonists to improve survival in patients with HFrEF. Our study showed that $94 \%$ of patients in the increased-dose group received ACEIs during discharge, compared with $96 \%$ of patients in the equal or reduceddose group. Beta blockers were prescribed in about $88 \%$ of patients in both groups. In addition, aldosterone antagonists were prescribed less commonly in the increased-dose group (71\%) compared with the equal or reduced-dose group (75\%). Finally, the use of hydralazine plus nitrates is recommended in recent guidelines for selected patients. Our study showed that roughly $56 \%$ of patients were on hydralazine plus nitrates in the increased-dose group compared with $57 \%$ in the equal or reduced-dose group. It should be noted that all patients included in our study received furosemide as the loop diuretic. The only explanation for this choice is the unavailability of torsemide in our institution and the availability of bumetanide for inpatients only.

Several limitations in our study should be mentioned. First, several medications commonly used in HFrEF were not available in our institution when the study was conducted. These include ivabradine, torsemide, and sacubitril/valsartan; thus, we could not evaluate their impact in our analysis. Second, we did not have any information regarding hemodynamic subsets based on fluid status and cardiac function, so the severity of ADHF was not considered in our study. Third, some of the baseline characteristics data were missing in the two groups, such as NTproBNP and follow-up serum creatinine during hospitalizations. Fourth, the all-cause readmission outcomes only were recognized in those patients readmitted to our institution; however, some patients might have been readmitted to other facilities. These readmissions could not be considered in our analysis given the absence of a shared health system database in our city. Finally, volume status, dietary intake, urine output, and weight changes were not retrievable from the system.

\section{CONCLUSIONS}

In conclusion, among patients admitted to the hospital with $\mathrm{ADHF}$ and reduced ejection fraction, a discharge loop 
diuretic dose that is higher than the home dose is associated with decreased all-cause 30-day, 60-day, and 90-day readmission rates. The results of this study highlight the benefit of increasing diuretic dose upon discharge in patients with $\mathrm{ADHF}$ and the need to perform larger randomized studies to confirm these findings.

\section{REFERENCES}

Arab News. 2018. http://www.arabnews.com/node/1259916/ saudi-arabia.

Brater D, Day B, Burdette A, Anderson S. 1984. Bumetanide and furosemide in heart failure. Kidney Int 26(2):183-9.

Brater DC. 2011. Update in diuretic therapy: clinical pharmacology. Semin Nephrol 31 (6):483-94.

Felker GM, Lee KL, Bull DA, et al. 2011. Diuretic strategies in patients with acute decompensated heart failure. N Engl J Med 364(9):797-805.

Fonarow GC, Stough G, Abraham WT, et al. 2007. Characteristics, treatments, and outcomes of patients with preserved systolic function hospitalized for heart failure. A report from the OPTIMIZE-HF Registry. J Am Coll Cardiol 50(8):768-777.

Go AS, Mozaffarian D, Roger VL, et al. 2013. Heart disease and stroke statistics--2013 update: a report from the American Heart Association. Circulation 127:e6-245.

Hasselblad V, Gattis Stough W, Shah MR, et al. 2007. Relation between dose of loop diuretics and outcomes in a heart failure population: results of the ESCAPE trial. Eur Heart J. 9(10):1064-1069.

Jovicic A, Holroyd-Leduc JM, Straus SE. 2006. Effects of self-management intervention on health outcomes of patients with heart failure: a systematic review of randomized controlled trials. BMC Cardiovasc Disord 6:43.

Kannel WB. 2000. Incidence and epidemiology of heart failure. Heart Fail Rev 5(2):167-73.

Kociol RD, Hammill BG, Fonarow GC, et al. 2010. Generalizability and longitudinal outcomes of a national heart failure clinical registry: Comparison of Acute Decompensated Heart Failure National Registry (ADHERE) and non-ADHERE Medicare beneficiaries. Am Heart J 160(5):885-92.

Krumholz HM, Merrill AR, Schone EM, et al. 2009. Patterns of hospital performance in acute myocardial infarction and heart failure 30-day mortality and readmission. Circ Cardiovasc Qual Outcomes 2(5):407-13.

MOH News 2013. https://www.moh.gov.sa/en/ministry/mediacenter/ news/pages/news-2013-10-30-002.aspx.

Publication Committee for the VMAC Investigators. 2002. Intravenous nesiritide vs nitroglycerin for treatment of decompensated congestive heart failure: a randomized controlled trial. JAMA 287(12):1531-40.

U.S. Department of Health and Human Services. 2011. The Booming Dynamics of Aging: From Awareness to Action. The White House Conference on Aging. Washington, DC: U.S. Department of Health and Human Services.

Woodruff AE, Kelley AM, Hempel CA, Loeffler WJ, Echtenkamp CA, Hassan AK. 2016. Discharge Diuretic Dose and 30-day Readmission Rate in Acute Decompensated Heart Failure. Annals of Pharmacotherapy 50(6):437-45.

Yancy CW, Jessup M, Bozkurt B, et al. 2013.2013 ACCF/AHA guideline for the management of heart failure: executive summary: a report of the American College of Cardiology Foundation/American Heart Association Task Force on practice guidelines. Circulation 128:1810. 